\title{
Germanica
}

les pays germanophones de 1880 à nos jours

\section{Periphere Räume erzählen}

Überlegungen zu narrativen Mustern am Beispiel des Unterlaufs der Donau

Narrer les espaces périphériques. Réflexions sur des modèles narratifs à

l'exemple du cours inférieur du Danube

How to describe peripheral areas? Reflections on narrative models on the basis of the representation of the lower Danube

\section{Olivia Spiridon}

\section{CpenEdition Journals}

Édition électronique

URL : http://journals.openedition.org/germanica/4217

DOI : 10.4000/germanica.4217

ISSN : 2107-0784

Éditeur

Université de Lille

Édition imprimée

Date de publication : 31 juillet 2018

Pagination : 19-31

ISBN : 9782913857414

ISSN : 0984-2632

Référence électronique

Olivia Spiridon, „Periphere Räume erzählen“, Germanica [Online], 62 | 2018, Online erschienen am: 31 Juli 2020, abgerufen am 15 Januar 2021. URL: http://journals.openedition.org/germanica/4217 ; DOI: https://doi.org/10.4000/germanica.4217 


\title{
Periphere Räume erzählen. Überlegungen zu narrativen Mustern am Beispiel des Unterlaufs der Donau
}

\author{
Olivia SPIRIDON \\ Institut für donauschwäbische Geschichte und Landeskunde
}

Flüsse implizieren Bewegung und Richtung, stellen Transportmöglichkeiten zur Verfügung und eignen sich dadurch als natürliche Medien von Reisen. Wie auch der Fall der Donau illustriert, wird ein Raum durchquert, der infolge sich wiederholender politischer Umformungen selbst in flüssigem Zustand ist. Darüber hinaus ist der Reisende in einem kulturellen Netz und einem soziopolitischen Kontext zu verorten, die die Wahrnehmung auf entscheidende Weise mitbestimmen. Am Wasserweg lagern sich zeitliche Sedimente $\mathrm{ab}$, sodass keine Reise wie die andere ist. Aus diesen Gründen mag es nicht verwundern, dass Franz Grillparzer auf der Reise von Wien nach Konstantinopel und Griechenland zwischen dem 27. August und dem 13. Oktober 1843, die er auf einem Dampfer donauabwärts unternommen hat, zwar bekannte Orte an der unteren Donau zur Kenntnis nimmt, wie Alt-Orschova, Medahia oder Küstentsche [Constanţa], sie jedoch in einem Zusammenhang wahrnimmt, der heute ohne weitere Erläuterung auf Unverständnis stößt.

Als Ausgangspunkt einiger einleitender Überlegungen sei ein Auszug angeführt, der seine Reise von der Donau über das Festland nach Constanţa beschreibt: 
Die Jagd so unglücklich als möglich. Schoß nur einmal, auf einen Pelikan, der zu hoch war und den ich daher verfehlte. Die Hunde schlecht. Die Rebhühner halten nicht aus. Verlieren uns endlich und kehre mit dem älteren Holländer allein nach dem Schiff zurück. Überall Wüste, nichts als Wüste. [...] Morgens um sieben Uhr zu Wagen weiter. Nirgends ein Dorf, höchstens Kirchhöfe als Überbleibsel von frühern. So fort durch zwanzig deutsche Meilen. Die Pferde, wo es möglich, in Galopp, ja in Karriere. Eine Reihe von Seen rechts am Wege, mit Wassergeflügel übersät. Nie in meinem Leben sah ich mehr Rebhühner beisammen. Geier, Habichte, auf alten Grabhügeln sitzend. Mitte Weges beim sogenannten Kaffeehause Streit mit einem Türken, dem sein Wagen erster Klasse zu schlecht ist, obwohl er nur für die dritte Klasse bezahlt. In der Nähe von Küstentsche Anblick auf das Schwarze Meer. Sieht aus wie ein dunkelblauer Hügel. Ostwind. Droht eine schlechte Überfahrt. Ankunft in Küstentsche. Zerstört wie alles Türkische ${ }^{1}$.

Doch wo ist Grillparzer zu verorten? Auch wenn er als Wiener Schriftsteller die Zensur in Österreich kennengelernt hatte ${ }^{2}$ und kein Freund der Zustände in der Habsburger Monarchie war, sah er darin, wenn auch nicht die Verwirklichung einer idealen Ordnung, so zumindest im Vergleich mit den von Revolutionen und Instabilität gebeutelten Nachbarländern ein real existierendes Potenzial. Politisch fühlte sich Grillparzer dem liberalen Bürgertum zugehörig, dessen Entfaltungsmöglichkeiten er in einer stabilen Ordnung sah, die seiner Ansicht nach der auf dem Naturrecht beruhenden monarchischen Staatsform entsprach ${ }^{3}$. In seinem Drama König Ottokars Glück und Ende, das 1825 in Wien uraufgeführt wurde, imaginierte er einen idealen Monarchen in einer Melange aus aufklärerischen Bausteinen und, zwecks Distanzierung, Mittelaltervorstellungen und bemühte darin die Donau als Band, das das Reich des aufgeklärten Monarchen zusammenhält: ,Schaut rings umher, wohin der Blick sich wendet,/Lachts wie dem Bräutigam die Braut entgegen! [...]/Ein voller Blumenstrauß so weit es reicht,/vom Silberband der Donau rings umwunden!‘4

1. - Franz Grillparzer, Tagebücher und Reiseberichte. Mit zeitgenössischen Illustrationen, hg. v. Klaus Geißler, Berlin, Verlag der Nation, 1980, S. 374.

2. - So zum Beispiel im Zusammenhang mit dem Drama König Ottokars Glück und Ende, 1825. Norbert Fürst, Grillparzer auf der Bühne. Eine fragmentarische Geschichte, Wien, München, Manutiuspresse, 1958, S. 100-102.

3. - Alfred Doppler, ,König Ottokars Glück und Ende. Das Verhältnis von dargestellter Zeit, Zeit der Darstellung und gegenwärtiger Rezeption“, in: Robert Pichl, Alexander Stillmark, Fred Wagner (Hg.), Grillparzer und die europäische Tradition, Wien, Hora, 1987, S. 22.

4. - Franz Grillparzer, König Ottokars Glück und Ende. Trauerspiel in fünf Aufzügen, hg. v. Helmut Bachmaier, Frankfurt a.M., Deutscher Klassiker Verlag, 1986, S. 458. 
Vor dem Hintergrund der politischen Anschauungen Grillparzers ist das oben angeführte Fragment seiner Reisebeschreibung zu deuten. Auffällig ist Grillparzers Blindheit für räumliche Details, die er zwar anführt (Ruinen der Kirchhöfe, Grabhügel, das Kaffeehaus), ohne jedoch die geringste Neugierde für die exotische Welt ringsum zu zeigen. Dafür wendet er seine Aufmerksamkeit voll und ganz den Türken und dem, was das Osmanische Reich repräsentiert, zu, und intensiviert die Wirkung seiner Wahrnehmung von wüsten, bedrohlichen Gegenden durch metaphorische Bilder - wie etwa der „Ostwind“ -, die den Leser unterschwellig unangenehm berühren. Seine Eindrücke erreichen schließlich bei der Ankunft in Konstantinopel und beim Anblick des Sultans inmitten seiner „lumpigen Truppen“ einen Höhepunkt ${ }^{5}$. Grillparzer nimmt die Donau als Verbindung und gleichzeitig Grenze zwischen zwei Reichen wahr, der Habsburger Monarchie und dem Osmanischen Reich, und ordnet auf seiner Reise durch osmanische Gebiete seine Wahrnehmung den Inhalten unter, die er in seinem Reisebericht im Grunde vermitteln möchte: die unmissverständlichen Zeichen des Verfalls des Osmanischen Reichs im Vergleich zum zivilisierten Habsburgerreich.

Mit Grillparzer sei ein Beispiel der politisch gelenkten Raumkonstruktion angeführt, die das Gebiet an der unteren Donau hervorbringt und den fließenden, unbeständigen Raum exemplarisch zeigt. Von Rumänien kann zu diesem Zeitpunkt noch nicht die Rede sein, sondern von dem an das Osmanische Reich angrenzende Fürstentum Walahei, denn die Staatsgründung erfolgt erst in der zweiten Hälfte des 19. Jahrhunderts. Grillparzers Reisebeschreibung stellt eine verhältnismäßig frühe Raumdarstellung der unteren Donau dar und fügt sich in ein Wahrnehmungsmuster ein, dessen Entwicklung in der zeitgenössischen Literatur an einem weiteren Beispiel verfolgt werden soll ${ }^{6}$.

Am Beispiel Grillparzer ist anhand der Modellierung der Donau und ihrer Umgebung durch die Darstellungsintention der Erzählinstanz der Konstruktionscharakter des Flusses und der Flusslandschaft nachzuvollziehen. Doch zunächst soll auf eine allgemeine Funktion von Landschaften und der ihnen innewohnenden Metaphorik hingewiesen werden. Landschaften können als „Modelle“ verwendet werden,

5. - F. Grillparzer, Tagebücher und Reiseberichte, a.a.O., S. 379.

6. - Aus Platzgründen sollen Betrachtungen der unteren Donau aus der Perspektive der Peripherie und ihrem Spannungsverhältnis zum Zentrum, so wie von Lotman angestellt und die sicherlich für diesen Grenzraum ergiebig ist, ausgeklammert werden. Siehe dazu: Jurij M. Lotman, ,Der Begriff der Grenze“, in: Susi K. Frank, Cornelia Ruhe und Alexander Schmitz (Hg.), Die Innenwelt des Denkens. Eine semiotische Theorie der Kultur, Berlin, Suhrkamp, 2010, S. 174-190; Albrecht Koschorke, Wahrheit und Erfindung. Grundzüge einer allgemeinen Erzähltheorie, Frankfurt a.M., S. Fischer, 2012, darin das Kapitel „Die Kultursemiotik Jurij Lotmans“, S. 116-128. 
,anhand derer differenzierte Aussagen über unterschiedliche soziale Zusammenhänge gemacht werden können"7. Mit seiner Reise an der Donau aus der Hauptstadt Habsburgs bis nach Konstantinopel als Herz des Osmanischen Reiches greift Grillparzer die den Flüssen immanente Metaphorik auf und visualisiert mit den Eindrücken vom Fluss und seinen Ufern abstrakte Geschichtsabläufe, um die es ihm im Grunde geht. In der Zeit nach den Türkenkriegen und der erfolgreichen Zurückdrängung der Osmanen durch die Habsburger, nach der Herausbildung der Donau der Habsburger als geopolitische Achse, eignet sich der Strom mit der ihm charakteristischen „Gerichtetheit“ und „Entschlossenheit zum Meer hin" 8 als Metapher der zivilisatorischen Mission, die die Habsburger Monarchie mit der Ausdehnung nach Südwesten verband. Die Reise flussabwärts impliziert zudem die Idee der Biografie und der Reifung 9 , die mit dem erfolgreichen Habsburgerreich aktiviert werden kann. $\mathrm{Zu}$ beachten ist, dass Grillparzer auf seinem Wasserweg den Blick nicht flussaufwärts richtet, was das Denken von Zweifeln und die Suche nach der Abstammung impliziert. Er schließt die im Rückblick unsichere Rekonstruktion des Flussverlaufs aus Hauptstrom und Zuflüssen sowie die nicht restlos geklärte Quelle von vornherein aus. Hinzu kommt auch die Ambivalenz des Flusses als Trennung und Verbindung: Das Band der Donau war jahrhundertelang von einer Grenze zwischen dem Heiligen Römischen Reich Deutscher Nation und dem Osmanischen Reich durchzogen, die Grillparzer stets in seinen Beobachtungen zur Rückständigkeit der Türken als kulturelle Grenze konstruiert. Allerdings schwingt mit der Grenze gleichzeitig das Bestreben ihrer Überwindung mit sowie die Chance der Verbindungsherstellung, die dem Strom innewohnt. Diese wurden in Grillparzers Drama über König Ottokar aufgegriffen und sie mussten in Grillparzers Wissenshorizont Anfang des 19. Jahrhunderts, in dem die erfolgreichen Türkenkriege und die habsburgische Raumpolitik präsent waren, einen festen Platz gehabt haben.

Aus seiner Reisebeschreibung geht hervor, dass er kaum an der Vermittlung von konkretem Raumwissen am unteren Donaulauf interessiert war. Dieser periphere Raum, der ein weißer Fleck in der mentalen Karte der damaligen Leser gewesen sein muss, eignete sich auch dank des dem Fluss inhärenten semantischen Potenzials für die Darstellung großflächiger Entwicklungen auf der politischen Karte Europas, und gerade das wurde von Grillparzer intensiv thematisiert. Dieses Beschreibungsmodell der Peripherie durch Ausblendung einer

7. - Rainer Guldin, Politische Landschaften. Zum Verhältnis von Raum und nationaler Identität, Bielefeld, transkript, 2014, S. 171. In diesem Zusammenhang ist insbesondere das Kapitel „Graben und Band. Landschaften als Modelle“von Relevanz.

8. - Ebd., S. 177.

9. - Ebd., S. 178. 
eingehenden Beschreibung der geografischen und Menschenlandschaft ist auch in anderen Texten des 19. Jahrhunderts anzutreffen, wie zum Beispiel in der in deutscher Sprache geschriebenen Beschreibung der Donaureise des Grafen István Széchenyi aus dem Jahr $1830^{10}$, der vor allem an der Schiffbarmachung des Stroms interessiert ist, in Mór Jókais Roman des künftigen Jahrhunderts ${ }^{11}$, in dem eine Stadt als heterotope Projektion an der Donaumündung imaginiert wird, oder in der fantasievollen Beschreibung der Donauvölker in Jules Vernes Roman Der Pilot von der Donau ${ }^{12}$, der seiner Faszination für die Befreiungskämpfe der Balkanvölker von den Türken freien Lauf lässt.

Um die Entwicklung von Beschreibungsmodellen der Peripherie am Beispiel des Unterlaufs der Donau in der Gegenwart zu illustrieren, wird ein Text hinzugezogen, der ebenfalls an einer Zeit- und Raumgrenze zu verorten ist. Eva Demskis kurz und bündig Mama Donau ${ }^{13}$ betitelte Erzählung oder Bericht über den Strom ist 2001 erschienen und ist, wie oft im Text durchscheint, aus der Erfahrung der Zeit nach dem Zerfall des Ostblocks und den Jugoslawienkriegen hervorgegangen. Dass das Buch den Strom räumlich in vertraute und weniger bekannte Segmente unterteilt, geht auch aus der zu Beginn abgedruckten Karte hervor, die im oberen Donauverlauf dicht mit Ortsnamen versehen und an der mittleren und unteren Donau zunehmend spärlicher beschriftet ist: in Ungarn sind lediglich Esztergom und Budapest verzeichnet und es folgen nach Belgrad keine Donaustädte mehr, lediglich Bukarest und Constanţa aus dem engeren Donauumfeld (vgl. Abbildung 1).

Die Formulierungen im Titel, „Mama Donau“, und auf dem Einband, „Ich bin an der Donau geboren... Das Buch erzählt meine Geschichten vom Fluss [...]", weisen auf eine von der Subjektivität einer Donauländerin gefärbte Erzählung mit autobiografischem Einschlag hin. Diese Erwartung wird bereits im Regensburger Teil bestätigt, in dem biografische Episoden in den Lebensraum der Stadt eingebettet werden, einer Welt, die sich aus der Wechselwirkung von Stadt und Strom zusammensetzt und deren Anschaulichkeit auch auf die Wiedergabe

10. - Gyula Viszota (Hg.), Gróf Széchenyi István Naplói [Tagebücher des Grafen István Széchenyi], Budapest, A Magy. Tud. Akadémia Hozzájárulásával Kiadja a Magyar Történelmi Társulat, 1934. Dabei handelt es sich um eine in ungarischer Sprache kommentierte Ausgabe des - mit Ausnahme einzelner französischer und ungarischer Sätze und Ausdrücke - deutschsprachigen Tagebuchs.

11. - Mór Jókai, Roman des künftigen Jahrhunderts, Pressburg und Leipzig, Verlag von Carl Stampfel, 1879.

12. - Jules Vernes Le Pilote du Danube erschien in Französisch und auch in deutscher Übersetzung im Jahr 1908.

13. - Die hier verwendete Textversion ist: Eva Demski, Mama Donau, Berlin, Insel, 2007. 


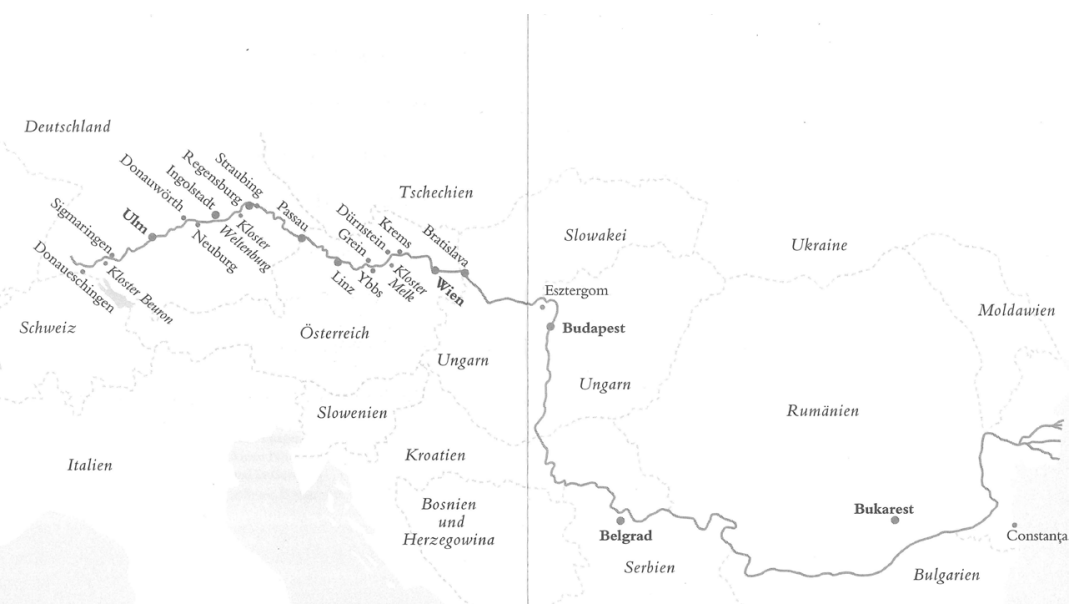

Abb. 1.

bayrischer Redensarten zurückzuführen ist, die lokale Praktiken sprachmalerisch wiedergeben. Im weiteren Erzählverlauf wird die Reiserichtung flussabwärts durch Reisen zu verschiedenen Zeitpunkten in der Vergangenheit verfolgt. Das Itinerarium von West nach Ost, die zeitliche Schichtung des Berichts und die Art des Erzählens verleihen dem Buch einen unmissverständlich politischen Charakter.

Budapest, wo die Narration einen ersten Halt im „Ostblock“ macht, befindet sich zeitlich und räumlich hinter dem Eisernen Vorhang, und dieser abgeschirmten und teilweise schwer zugänglichen Welt wird durch das Hervorheben zahlreicher Verständnisschwierigkeiten begegnet: die Existenz von nur für westliche Touristen zugänglichen Hotels, die soziale Kluft, die sich in Ländern der Gleichheitsideologen aufgetan hat, die Überwachung durch den Geheimdienst, der durch einen getarnten Dolmetscher die Kommunikation zwischen Einheimischen und Besuchern erschwert, der Besuch politischer Dissidenten in der Gestalt des Schriftstellers Tibor Déry, die Verwunderung über die außergewöhnliche Kaufkraft der westlichen Währung usw. Budapest markiert einen Moment des Zweifels über die Existenz des Stroms als Band, der die Lebenswelt aus dem Mittellauf mit jenen des Oberlaufs zu verbinden vermag: „Unten lag meine Donau und spiegelte die funzligen Lichter, die sich Budapest damals leisten konnte. Sogar sie war mir fremd. Wenn ich, dachte ich, jetzt ein Schiff bekäme und flussaufwärts fahren würde? Ich war nicht mehr sicher, ob ich in Regensburg ankäme." ${ }^{14}$ An einer anderen Stelle der Reisebeschreibung durch Ungarn 
wird ein Gegeneinander der Kräfte geschildert, der flussaufwärts strebenden Schwimmerin und des abwärts strömenden Flusses. Aus diesem Unsicherheit ausdrückenden Moment der gleichzeitigen Auf- und Abwärtsbewegung wird die Raumwahrnehmung gesteuert, wobei in Demskis Darstellung die Weite des Raums und die Entschlossenheit der Flussrichtung die entscheidenden Vektoren sind. Sie bringen das Bild der Donau als ein Streben nach der Mündung hervor: „Ich schwimme, ich schwimme, aber sie bestimmt die Richtung.“ (MD, 126)

Nach einem Ausflug in die ungarische Puszta erfolgt die Rückreise mit der „Donauprinzessin“ nach Deutschland, denn Richtung Delta fährt das Schiff nicht und „das Flusshandbuch wird für diesen Teil erstmal nicht nachgedruckt.“ (MD, 127) Nur noch ein Kapitel enthält die Erzählung Demskis, „Die Prinzessin fährt nicht nach Constanţa“ (MD, 127-136), das mit dem Bedauern einsetzt, dass die Reise „ein Fragment geblieben ist" (MD, 127), und eine Beschreibung Südrumäniens und der Hauptstadt von Bulgarien beinhaltet.

Neben den inhaltlichen Schwerpunkten des Buches ist die Konzeption der Narration von Bedeutung. Zu Beginn, im Regensburger Teil, am Rande der aus verschiedenen narrativen Mosaikstücken zusammengefügten Erzählung von Stadt und Strom, kommt es zu einer Analogie zwischen dem Fluss und dem Erzählfluss: „,...] - und jetzt geht die Geschichte wieder auseinander, bildet gleichsam Altarme und stille Stellen.“ (MD, 17) Erst in Verbindung mit diesem Schlüssel zum Aufbau der Narration erschließt sich dem Leser der Sinn dieser auf den ersten Blick unausgewogenen Darstellung der Donau, die schon angesichts der ungleichmäßig beschrifteten Karte des Stroms auffällt. So ist das Rumänien-Kapitel durch fehlende Schärfe und ungenaue Angaben gekennzeichnet, die - würde sich die Wahrnehmung des Lesers nicht auf die Folie des Strommodells als Verkörperung des Unübersichtlichen und der Unordnung legen - an Schlamperei denken ließe. Doch nun scheint dieses narrative Konzept im Zusammenhang mit der Aussage an anderer Stellte, dass das Delta der Raum der „Auflösung des Stroms in seine große Unordnung" darstelle (MD, 109), programmatisch: „Bukarest liegt an der Grenze. Die Donau ist die Grenze. Da, wo ich herkam, gehörten mir beide Ufer. Trotzdem war mir etwas an Bukarest vertraut, weil es eine Donaustadt ist. Ich kann nicht sagen, was es war.“ (MD, 128) Die Reihe der Ungereimtheiten setzt sich fort, als die Zeitangabe der Reise, das Jahr 1971, als der ,kurze rumänische Frühling Anfang der siebziger Jahre“ (MD, 129) bezeichnet wird. Der an den Prager Frühling angelehnte Begriff trifft jedoch für diese Zeit nicht mehr zu, denn 1971 wurde die Mitte der 1960er 
Jahre eingeleitete Liberalisierungsphase mit den „Juli-Thesen“15 des Staatspräsidenten Ceauşescu aufgekündigt und es folgte eine sich stetig ausbreitende Eiszeit bis zur politischen Wende 1989. Die Unordnung der Chronologie kennzeichnet den letzten Teil des Buches, denn die Ungarnreise, die zum Zeitpunkt des dreiunddreißigsten Geburtstags der Erzählerin stattgefunden hat (MD, 105) und auf die zweite Hälfte der 1970er Jahre zu datieren ist, wird dem Rumänienkapitel vorangestellt und die unvollständige Befahrung des Stroms ab Budapest Richtung Delta bedauert (MD, 127), obwohl die Rumänienreise mit integriertem Besuch des Donaudeltas bereits sechs Jahre früher stattgefunden hat, wie aus dem anschließenden und letzten Kapitel hervorgeht. Es entsteht der Eindruck, dass die Erzählinstanz darauf abzielt, Verwirrung zu stiften und die Unmöglichkeit der Durchdringung dieser erzählten Welt zu veranschaulichen, da sich die Realität des Landes der Besucherin auf weiten Strecken entzieht und diese gestehen muss, im Grunde über etwas Irreales zu berichten:

Ich fand Bukarest eine wunderbare Stadt, etwas grau überhaucht, in der Nacht fegten Frauen die Straßen, und das Hotel Athenée Palace später, viel später ein Schauplatz für die Orgien des Ceausescu-Sohnes - glomm prachtvoll vor sich hin im Schein der Zwanzigwattbirnen. Es war überall entsetzlich heiß, das kannten wir aus dem verschwendungssüchtigen Kapitalismus nicht. Hier rissen wir die Fenster auf und wunderten uns über alles und gar nichts. Wie viele Minister es gab. Andauernd waren wir zu Empfängen bei Ministern eingeladen. Gott schütze unsere jugendlichen Lebern, die sich an warmen Zuica zum Frühstück gewöhnen mußten, eiskalten Champagner zu Mittag, schwere, ölige Brände aus Georgien, süße Dessertweine, wenn ich mich recht erinnere. Wir waren alle in einem Zustand trügerischer Wachheit. Nachts verließen wir das Hotel, um die Stadt zu entdecken. Und tatsächlich folgten uns Männer mit langen Mänteln und Hüten. Wir waren entzückt - ein Film, ganz für uns allein! Die Wirklichkeit könnte das ja nicht sein. (MD, 127-128)

Es ist nicht nur, dass der Erzählerblick die Wasserstraße verlässt und über den weiteren Donauraum ausschweift, indem neben der kurzen Beschreibung des Aufenthalts in Bukarest im gleichen abschlie-

15. - Cristina Petrescu, Dragoş Petrescu, „,Tezele restalinizării culturale româneşti - iulie 1971“ [,Die Thesen der kulturellen Restalinisierung in Rumänien - Juli 1971“], in: Cotidianul. Supliment de istorie vom 25.10.1996, S. 1-3. Die 17 Thesen Ceauşescus zur ,Verbesserung der politisch-ideologischen Arbeit und der kulturellen und erzieherischen Tätigkeit" legen vom Einfluss des chinesischen und nordkoreanischen Staatsmodells auf den Präsidenten Rumäniens Zeugnis ab und wurden am 6. Juli 1971 vom Exekutivkomitee der rumänischen kommunistischen Partei verabschiedet. Sie festigten die Hegemonie der Partei, sicherten ihre direkte Leitung, die totale Ideologisierung der Kultur, Wissenschaft und Erziehung. 
Benden Kapitel auch die bulgarische Hauptstadt Sofia geschildert wird. Die Donau selbst kommt nur spärlich, in einer Reihe allgemeiner Feststellungen zur Sprache: „Ich erinnere mich an unglaublich viele Mücken, eine Landschaft, viel zu groß und unübersichtlich, als daß Politik ihr etwas anhaben könnte, Wälder aus Schilf, große Vögel. [...] Das Donaudelta ist das Land meiner Sehnsucht. [...] Die stille Internationale der Ornithologen findet dort ein Gelobtes Land [...]“. (MD, 130)

Doch nun mäandert Demskis Text durch andere Texte, was mit der nebulösen Feststellung eingeleitet wird, dass „dies Kapitel“ „,aus sehr ferner Vergangenheit und hoffentlich naher Zukunft" „gemacht" sei (MD, 130-131). Als Ersatz für eine Donaubeschreibung aus eigener Sicht wird nun Lothar-Günther Buchheims Reise mit einem Faltboot ${ }^{16}$ in einigen Absätzen wiedergegeben, der Reiseverlauf von Passau bis zum Schwarzen Meer gezeichnet und die Publikation des Buches „1939 bei S. Fischer" (MD, 139) als editorisches Ereignis hervorgehoben. Das Besondere dieses Reiseberichts über die Vielfalt der Lebenswelten am Strom ist nämlich seine Veröffentlichung in einer Zeit, als die Donau gerade durch Ausblendung ihrer Mannigfaltigkeit in die nationalsozialistische Raumstrategie eingebunden wurde ${ }^{17}$. Doch auch hier schleichen sich Ungenauigkeiten ein: Die Reise fand 1938 und nicht 1937 statt, wie bei Demski angeführt, und die Erstveröffentlichung erfolgte nicht 1939, sondern zwei Jahre später.

Demskis Raumdarstellung entsteht durch Verflechtung mit anderen Texten, die den Raum entweder aus der Außenperspektive wahrnehmen, wie das Beispiel Buchheim zeigt, oder aus der rumänischen Perspektive. Von Buchheim wird der Eindruck der Hybridität Bukarests übernommen, das eine „Mischung aus Marodem und entschlossener Modernität“, „wie Detroit oder Michigan“18 sei. Dem fügt Demski eigene Erfahrungen von ihrem Aufenthalt als junge Journalistin aus dem Westen hinzu, die von hohen Parteifunktionären umworben wurde. Sie erlebt eine Welt der Gegensätze, so etwa barfüßige alte Frauen als Teil des Stadtbildes und üppige Empfänge bei verschiedenen Ministerien, wo sie über mit Kaviar gefüllte Eisschwäne staunt. Darüber hinaus bekommt die Erzählerin von einem Kenner des Landes, einem nicht

16. - Lothar-Günther Buchheim, Tage und Nächte steigen aus dem Strom, München, Langen Müller, 2008.

17. - Vgl. dazu Victor Pietschmann, Die Donau: Deutschlands anderer Schicksalsstrom, Jena, Diederichs, 1938; eine Übersicht über die Donau in der nationalsozialistischen Ära liefert Ortrun Veichtlbauer, ,Braune Donau. Transportweg nationalsozialistischer Biopolitik“, in: Christian Reder, Erich Klein (Hg.), Graue Donau, Schwarzes Meer, Wien, Springer, 2008, S. 226-245.

18. - Diese Passagen aus Mama Donau findet man in der Ausgabe von Buchheim von 2008, S. 214. 
beim Namen genannten ,großartigen rumänischen Dichter“, Einblicke aus der Innenperspektive in die Mentalität des Landes, in dem der Neid regiert, sodass der Misserfolg des anderen mehr zählt als der eigene Erfolg (MD, 132).

An solchen Stellen ist nachzuvollziehen, wie der Text über diesen peripheren europäischen Raum durch Wissenstransfer aus anderen Quellen entsteht, und wie Verwunderung und das Nicht-Verstehen zu einem festen Bestandteil der Darstellung werden. Dies wird auch in einem anderen Essay von Eva Demski über Rumänien und seine Donaulandschaft sichtbar, in dem von einer Autoreise mit dem Dichter Mircea Dinescu von Bukarest nach Cetate, einem ehemaligen Donauhafen, berichtet wird, der von Dinescu zu einem Treffpunkt für Künstler ausgebaut wurde ${ }^{19}$. Unterwegs wird in einem „Zigeunerdorf“ haltgemacht, einem Zeugnis der „steingewordenen Erfolgsgeschichte“ der Minderheit der Roma, die Demski bis dahin nur aus den ärmlichen Siedlungen am Rande Bukarests kannte.

Wir waren langsam an der Kirche vorbeigefahren und standen nun auf einer Dorfstraße, wie ich nie im Leben eine gesehen hatte. Rechts und links von ihr - was waren das für Häuser? Schlösser? Burgen? Alpträume? Hatte in dieser gottverlassenen Gegend ein DisneylandBühnenbildner Drogenfantasien verwirklicht? War diese Straße ein Wunder oder ein Grauen? Ich hielt den Mund und staunte [...] Keines der Häuser glich seinem Nachbarn, und dennoch trugen sie alle die gleiche gestalterische Handschrift oder folgten dem gleichen Wahn: Mauern in Silber, bedeckt mit dunklen Zeichen und Mustern, Türmchen, Erker und Zinnen, die Türme trugen goldene Spitzen. Manche hatten Portale und Säulen, es gab komplizierte Dachformen und seltsam geformte Traufen, aber alles sah wie ein Bühnenbild aus oder wie eine Kulissengasse für, ja, was? Welche Art Film könnte hier gedreht werden? ${ }^{20}$

Neben der unvorstellbar fremden Welt der Roma, später auch der anschließend besuchten Lipowaner, einer russischsprachigen Minderheit von im Donaudelta angesiedelten Altgläubigen, wird wieder ein Bild der Donau eingeblendet: „Klar und breit war sie, nichts erinnerte mehr an den dreckigen, geschäftigen Strom von einst [...]“21.

Aus der Betrachtung der beiden Texte ist die Motivation der Autorin, über die Donau und den Donauraum zu schreiben, nachzuvollziehen, die sich nicht aus sachlichem Interesse für eine europäische Großregion nährt und auch über das oberflächlich Politische hinausgeht. Ihr Movens

19. - Eva Demski, ,Sie nennen sich Rom, das heißt Mensch. Mit dem rumänischen Dichterfürsten Mircea Dinescu in einer Zigeunerstadt aus Silber und Gold“", in: du. Zeitschrift für Kultur Nr. 777 (2007-2008), S. 58-62.

20. - Ebd., S. 58.

21. - Ebd., S. 59. 
wird in der Liebeserklärung für einen Fluss sichtbar, der im Ober- und Unterlauf so verschiedenartige Natur- und Menschenlandschaften durchquert: „Was aber blieb, jenseits politischer und ideologischer Wankelmütigkeit - jedenfalls war und ist das bei mir so: die Sehnsucht nach dem Wilden, dem Ungeregelten, dem Ungezähmten. Je langsamer und träger die Donau wird, müde von ihrem langen Weg, ausruhend in der ungarischen Tiefebene und ihren Verzweigungen - desto wilder und heftiger geht es an ihren Ufern zu.“ (MD, 133)

Das Ungeregelte und die bereits erwähnte Zunahme von Unordnung am Unterlauf des Stroms finden inhaltlich in Unschärfe, Ungereimtheiten und Unverständnis ihren Niederschlag. Stilistisch wird das Wasser mit dem durcheinander fließenden Treibgut, den Seitenarmen und der fehlenden Übersichtlichkeit zum Modell, das sich auf das Gewebe des Textes von Beginn an überträgt. Unpassendes steht nebeneinander und die Juxtaposition ist ein häufig eingesetztes Ausdrucksmittel: In Regensburg „schlürft“ die Donau badende Kinder ,ein“ (MD, 11), in der Stadt gibt es „blutige, stinkende, wunderbare Märkte“ (MD, 17), Budapest ist eine ,zu Tode erschöpfte und lustige graue Stadt" (MD, 113). Für den mittleren und unteren Stromlauf wird auf eine Aufzählung Buchheims zurückgegriffen, die den Raum als Entfaltungsort von Heterogenem zeichnet: „Ich habe allerlei Vorstellungen von starkem Kaffee, Steppenvieh, Paprika, Wasserpfeife, Allah-ist-groß, Zigeunermusik, Ferkel am Spieß, Rosenfeldern, halbwilden Gebieten und hypermodernen Hochhäusern zwischen patriarchalischen Holzhütten -, und nun will ich einmal nachschauen, was es damit auf sich hat." (MD, 131)

Das Beschreibungsmodell des Unterlaufs der Donau durch Ungenauigkeiten, Auslassungen und Aufnahme von Ersatztexten zum Ausfüllen von Wissens- und Erfahrungslücken hat Tradition. Der Richtung Flussmündung knapp werdende Text Eva Demskis erinnert an die Donaubeschreibung von Siegmund von Birken aus dem 17. Jahrhundert ${ }^{22}$. In der Vergangenheit waren die Unwegbarkeit des Stroms vor allem nach dem Eisernen Tor ein Grund für die spärlichen Texte über den unteren Donaulauf, die Länge und Schwierigkeit des Wasserwegs, die so manch einer geplanten Reise und Publikation, wie im Fall von Johann Georg Kohl und später von Algernon Blackwood, frühzeitig ein Ende setzten ${ }^{23}$. Ab Mitte des 20. Jahrhunderts waren es

22. - Siegmund von Birken, Der Donau-Strand: mit Allen seinen Ein- und Zuflüssen, angelegenen Königreichen, Provinzen, Herrschafften und Städten ... vom Ursprung biß zum Ausfluss: in Dreyfacher Land-Mappe vorgestellet auch samt kurzter Verfassung einer Hungar- und Türckisischen Chronik, und des Anno 1663 und 1664 geführten Türken-Krieges, Nürnberg, 1683.

23. - Johann Georg Kohls Die Donau von ihrem Ursprunge bis Pesth erschien 1854, doch die geplante Fortsetzung des Donau-Werks bis Konstantinopel blieb aus nicht ganz geklärten Gründen aus. Thomas Elsmann, Johann Georg Kohl. Ein Leben zwischen 
die politische Teilung Europas bis 1989 und in den 1990er Jahren die Jugoslawienkriege und die Brückenruinen die Hindernisse, die den Zugang zur Strommündung erschwerten.

Musterbildend ist auch das Spannungsverhältnis, das aus der Ambivalenz des Stroms hervorgeht, der sowohl die Verbindung durch das natürliche „Band“ als auch die Trennung durch hegemoniale Ansprüche versinnbildlicht. Bei Grillparzer fungierte die Donaureise als Anlass für die Inszenierung eines europäischen politischen "Schachbretts“ mit festen Zuschreibungen - Habsburg-Fortschritt versus Osmanenzivilisatorischer Verfall -, wobei Grenzen im Zusammenhang mit ihrer Überwindung durch die Öffnung Habsburgs nach Südosten gedacht wurden. Gleichzeitig ist Grillparzers Donaureise sowie auch die Zeichnung einer politischen Landschaft Österreichs mit dem Band der Donau als zentralem Landschaftsmerkmal im Drama König Ottokars Glück und Ende als persönliches Statement der Zugehörigkeit zur Habsburger Monarchie und zur stabilen Ordnung anzusehen, die sie repräsentierte. Auch für Demski stellt die Beschreibung des Unterlaufs eine Möglichkeit dar, sich als Subjekt auf einer politischen Karte zu positionieren. Nach der politischen Wende 1989 wird eine Landschaft durch die Reise wieder zu einem - wenn auch heterogenen - Ganzen zusammengefügt, das im geteilten Europa auseinandergerissen war. Im Unterschied zu Grillparzer, dessen Faszination in erster Linie der Zeit und historischen Abläufen gilt, interessiert sich Demski für den Raum - und da fügt sich ihre Beschreibung in ein aktuelles Wahrnehmungsschema ein, das dem Räumlichen Priorität über das Zeitliche gibt. Die Reise erfolgt nicht linear, in eine Richtung, wie bei Grillparzer, sondern besteht aus mehreren, nicht chronologisch geordneten Reisen, und die Beschreibung hebt miteinander vernetzte Punkte sowie Verbindungen zwischen ihnen hervor, sodass der Donauraum den Eindruck eines lebendigen Netzwerks voller Unstimmigkeiten, Widersprüche, Brüche, aber auch Kontinuitäten macht ${ }^{24}$. Zudem veran-

der alten und der neuen Welt, Bremen, Carl Schünemann, 2010, S. 129-130. Algernon Blackwood, „Eine Kanufahrt auf der Donau“, in: Ders., Die Weiden. Eine phantastische Geschichte. Aus dem Englischen von Friedrich Polakovics. Eine Kanufahrt auf der Donau. Ein Reisebericht. Aus dem Englischen von Melanie Walz, Frankfurt a.M., Heinrich und Hahn, 2007.

24. - In diesem Sinn argumentiert auch Karl-Markus Gauß im Essay „Die Lehre der Donau“ in einem Satz voller Zuflüsse, wie auch der Strom selbst: „Was die Donau mit ihren Ländern und Menschen so anziehend macht, ist eben die Gleichzeitigkeit, der sich auf engem Raum entfaltende Widerspruch: nicht unberührte Natur, nicht einheitlich durchformte Kultur, bietet die Donau immer das Zugleich, von Völkern, Religionen, Sprachen, von Entwicklungsstufen der Ökonomie, von Traditionen, die nicht preisgegeben, von Aufbrüchen, die gewagt werden, von Besonderheiten einer Volksgruppe, die trotzig bewahrt, und Verflechtungen mit der Welt, die als das Selbstverständliche gesucht werden." In: Inge Morath, Donau. Mit einem Essay von Karl-Markus Gauß, Salzburg, 
lasst der Strom in Eva Demskis Mama Donau die Selbstdefinierung von Identität durch Bejahung von Diversität, Akzeptanz des Nicht-VerstehenKönnens und die Erweiterung des persönlichen Geborgenheitsraums: „Das Donaudelta ist das Land meiner Sehnsucht.“ (MD, 130) Die Donau repräsentiert die Chance, eigene Eigenschaften, ,die Sehnsucht nach Wildem, Ungeregeltem, Ungezähmtem“ (MD, 133), durch Analogien zum mächtigen Wasserlauf des Stroms zu legitimieren und aufzuwerten. Doch, über alle Differenzen hinaus, fungiert die Donau in Texten aus verschiedenen Jahrhunderten als Kulisse subjektiver Befindlichkeiten, und die Wahrnehmung des Unterlaufs des Stroms spiegelt sich im Gewebe der Donau-Texte wider. 
